\title{
Continuous-variable phase estimation with unitary and random linear disturbance
}

\author{
Douglas Delgado de Souza, ${ }^{1,2}$ Marco G. Genoni, ${ }^{3}$ and M. S. Kim ${ }^{2}$ \\ ${ }^{1}$ Instituto de Fisica Gleb Wataghin, Universidade Estadual de Campinas, 13083-970, Campinas, SP, Brazil \\ ${ }^{2}$ QOLS, Blackett Laboratory, Imperial College London, London SW7 2BW, United Kingdom \\ ${ }^{3}$ Department of Physics \& Astronomy, University College London, Gower Street, London WC1E 6BT, United Kingdom
}

(Received 31 July 2014; published 27 October 2014)

\begin{abstract}
We address the problem of continuous-variable quantum phase estimation in the presence of linear disturbance at the Hamiltonian level by means of Gaussian probe states. In particular we discuss both unitary and random disturbance by considering the parameter which characterizes the unwanted linear term present in the Hamiltonian as fixed (unitary disturbance) or random with a given probability distribution (random disturbance). We derive the optimal input Gaussian states at fixed energy, maximizing the quantum Fisher information over the squeezing angle and the squeezing energy fraction, and we discuss the scaling of the quantum Fisher information in terms of the output number of photons, $n_{\text {out }}$. We observe that, in the case of unitary disturbance, the optimal state is a squeezed vacuum state and the quadratic scaling is conserved. As regards the random disturbance, we observe that the optimal squeezing fraction may not be equal to one and, for any nonzero value of the noise parameter, the quantum Fisher information scales linearly with the average number of photons. Finally, we discuss the performance of homodyne measurement by comparing the achievable precision with the ultimate limit imposed by the quantum Cramér-Rao bound.
\end{abstract}

DOI: 10.1103/PhysRevA.90.042119

PACS number(s): 03.65.Wj, 03.65.Ta, 42.50.Dv

\section{INTRODUCTION}

The usefulness of the nonclassical features of quantum mechanics to perform ultraprecise measurement beyond the classical limit has recently indicated quantum metrology as one of the most promising quantum technologies [1]. Phase estimation is the paradigmatic example of an estimation problem, and the possible quantum enhancement has been widely studied both theoretically and experimentally [2-11]. The role of noise in quantum metrology was first investigated in Ref. [12]: there it was shown how the quantum enhancement in frequency estimation is lost if one considers the system interacting with a dephasing environment. These and other results were rigorously confirmed in Refs. [13,14] where it was proved that, when the noisy dynamics is ruled by a semigroup, the ultimate quantum limit is lost and only a constant enhancement over the standard quantum limit may be obtained. However attempts to circumvent these no-go theorems have been already presented by considering frequency estimation with specific noisy evolutions [15] or by implementing error-correcting codes [16-18].

While in the case of frequency estimation $[12,19]$ the phase rotation and the noise are treated simultaneously by using a master-equation approach; for phase estimation typically one considers the situation where the phase rotation is performed on the initially pure probe state, and the noisy channel is applied afterwards on the encoded states. By following this approach, the role of loss [20-23] and phase diffusion [24-30] were investigated in great detail. However, one can also consider the case where an unwanted but known and fixed term is present in the Hamiltonian generating the phase rotation, influencing the estimation process. This problem was studied for the first time in Ref. [31] by De Pasquale and coauthors who referred to it as unitary disturbance. One can then consider a more general, and probably more realistic case, where the disturbance parameter characterizing the additional term in the Hamiltonian is a random variable distributed according to a known probability distribution. We will refer to this case as random disturbance.

In this paper we consider continuous-variable phase estimation with both unitary and random disturbance, where the additional term in the Hamiltonian is linear in the bosonic operators describing the quantum system under examination. Ideal phase estimation with Gaussian probe states was first investigated in Ref. [32]; it was demonstrated that squeezed states are optimal, and that the corresponding quantum Fisher information (QFI) scales quadratically with the average number of photons, showing the enhancement compared to the classical linear scaling obtainable with coherent states. Here we will derive the QFI both for unitary and random linear disturbance, finding the influence of the noise parameters on the optimal input Gaussian states, and on the corresponding scaling between the QFI and the output states' average number of photons. The manuscript is organized as follows: in Sec. II we introduce quantum estimation theory, along with the formulas for the QFI in the case of unitary disturbance and for generic Gaussian states. In Secs. III and IV we present the result concerning the optimal QFI and the optimal probe states for the unitary- and randomdisturbance cases, respectively. At the end of both sections we discuss the precision achievable via homodyne detection in the relevant cases, comparing it with the ultimate bounds just derived. Section V ends the paper with some concluding remarks.

\section{QUANTUM ESTIMATION THEORY}

Let us consider a family of quantum states $\varrho_{\phi}$, where $\phi$ is the parameter one wants to estimate. A measurement, parametrized by positive operator valued measure (POVM) operators $\left\{\Pi_{x}\right\}$, can be fully described by means of the conditional probability distribution $p(x \mid \phi)=\operatorname{Tr}\left[\varrho_{\phi} \Pi_{x}\right]$. The corresponding precision on the estimation of the parameter $\phi$, 
by means of the measurement $\left\{\Pi_{x}\right\}$ is bounded as

$$
\delta \phi \geqslant \frac{1}{M F(\phi)},
$$

where $M$ is the number of measurements and

$$
F(\phi)=\int d x p(x \mid \phi)\left[\partial_{\phi} \ln p(x \mid \phi)\right]^{2}
$$

is the classical Fisher information (FI). The inequality (1) is called Cramér-Rao bound (CRB), and it holds for every classical estimation problem described by a conditional probability $p(x \mid \phi)$. The bound is achievable by means of maximum likelihood and Bayesian estimators in the limit of a large number of measurements.

By considering the quantum case and defining the symmetric logarithmic derivative operator $L_{\phi}$ by means of the equation

$$
2 \partial_{\phi} \varrho_{\phi}=L_{\phi} \varrho_{\phi}+\varrho_{\phi} L_{\phi},
$$

it is possible to demonstrate that the FI, for any POVM, is bounded from above as

$$
F(\phi) \leqslant H(\phi),
$$

where $H(\phi)=\operatorname{Tr}\left[\varrho_{\phi} L_{\phi}^{2}\right]$ is the QFI $[33,34]$. This inequality leads to the quantum Cramér-Rao bound (QCRB)

$$
\delta \phi \geqslant \frac{1}{M H(\phi)} .
$$

It can be demonstrated that this bound is always in principle achievable; that is, there is always a POVM whose corresponding classical FI is equal to the QFI. It is then clear by observing Eq. (5) that a larger value of the QFI corresponds to a higher precision achievable by means of the encoded state $\varrho_{\phi}$.

\section{A. Quantum estimation with unitary disturbance}

Let us first consider the case of quantum estimation of unitary parameters. If the parameter is encoded via a unitary operation with a Hermitian generator $G$,

$$
\varrho_{\phi}=U_{\phi} \varrho_{0} U_{\phi}^{\dagger}, \quad U_{\phi}=e^{-i \phi G},
$$

and the probe state $\varrho_{0}=\left|\psi_{0}\right\rangle\left\langle\psi_{0}\right|$ is pure, the QFI can be easily evaluated as

$$
H(\phi)=4 \Delta^{2} G=4\left(\left\langle\psi_{0}\left|G^{2}\right| \psi_{0}\right\rangle-\left\langle\psi_{0}|G| \psi_{0}\right\rangle^{2}\right) .
$$

One can rather consider the case where an additional term is present in the generator of the unitary interaction encoding the parameter $\phi$, i.e., when the unitary operation reads

$$
U_{\phi, \eta}=\exp \{-i H(\phi, \eta)\}:=\exp \{-i(\phi G+\eta A)\},
$$

where $\eta \in \mathbb{R}$ is a fixed noise parameter and $A$ is the additional disturbance (Hermitian) operator. In Ref. [31], the authors addressed this general problem and derived the following formula to calculate the QFI:

$$
H(\phi)=4 \Delta^{2} \bar{G}(\phi, \eta),
$$

where

$$
\bar{G}(\phi, \eta)=\int_{0}^{1} d t e^{i H(\phi, \eta) t} G e^{-i H(\phi, \eta) t}
$$

\section{B. Quantum estimation with Gaussian states}

Let us consider a quantum system described by bosonic operators $\left[a, a^{\dagger}\right]=\mathbb{1}$. A quantum state $\varrho$ can be fully described by its characteristic function $\chi[\varrho](\alpha)=\operatorname{Tr}[\varrho D(\alpha)]$, where $D(\alpha)=\exp \left\{\alpha a^{\dagger}-\alpha^{*} a\right\}$ is the displacement operator in phase space. If the characteristic function $\chi[\varrho](\alpha)$ is a Gaussian function, the state is said to be Gaussian [35]. By defining the quadrature operators vector $\mathbf{X}=(Q, P)^{T}$, where

$$
Q=a+a^{\dagger}, \quad P=-i\left(a-a^{\dagger}\right)
$$

the Gaussian quantum state can be fully described by the corresponding average values $\overline{\mathbf{X}}$ and the covariance matrix $\boldsymbol{\sigma}$, defined as

$$
\begin{aligned}
\bar{X}_{j} & =\left\langle\psi_{0}\left|X_{j}\right| \psi_{0}\right\rangle, \\
\sigma_{j k} & =\frac{1}{2}\left\langle\psi_{0}\left|X_{j} X_{k}+X_{k} X_{j}\right| \psi_{0}\right\rangle-\bar{X}_{j} \bar{X}_{k} .
\end{aligned}
$$

If we are considering an estimation problem where the quantum state $\varrho_{\phi}$ is Gaussian, one can derive closed formulas for the QFI in terms of the vector $\overline{\mathbf{X}}_{\phi}$ and the matrix $\sigma_{\phi}$ only [36,37], obtaining

$$
H(\phi)=\frac{1}{2} \frac{\operatorname{Tr}\left[\left(\sigma_{\phi}^{-1} \boldsymbol{\sigma}_{\phi}^{\prime}\right)^{2}\right]}{1+\mu_{\phi}^{2}}+2 \frac{\left(\mu_{\phi}^{\prime}\right)^{2}}{1-\mu_{\phi}^{4}}+\Delta \overline{\mathbf{X}}_{\phi}^{\prime \top} \boldsymbol{\sigma}_{\phi}^{-1} \Delta \overline{\mathbf{X}}_{\phi}^{\prime}
$$

In the formula, $\mu_{\phi}=\operatorname{Tr}\left[\varrho_{\phi}^{2}\right]=1 / \sqrt{\operatorname{det}[\sigma]}$ represents the purity of the state, primed quantities corresponds to derivatives with respect to the parameter $\phi$, except for $\Delta \overline{\mathbf{X}}_{\phi}^{\prime}$ which is defined as

$$
\Delta \overline{\mathbf{X}}_{\phi}^{\prime}=\left.\frac{d\left(\overline{\mathbf{X}}_{\phi+\epsilon}-\overline{\mathbf{X}}_{\phi}\right)}{d \epsilon}\right|_{\epsilon=0} .
$$

This expression will be extremely useful to analytically calculate the QFI for the random linear disturbance in Sec. IV.

\section{UNITARY LINEAR DISTURBANCE}

In the following we study the problem of phase estimation with unitary linear disturbance, by considering the unitary operator in Eq. (8) where the generator and the disturbance operator read, respectively,

$$
G=a^{\dagger} a, \quad A=Q=a+a^{\dagger},
$$

such that

$$
U_{\phi, \eta}=\exp \left\{-i\left[\phi a^{\dagger} a+\eta\left(a+a^{\dagger}\right)\right]\right\}
$$

Its effect is a phase rotation accompanied by a displacement in phase space; however, because the operators $G$ and $D$ do not commute, the two effects cannot be separated. We are interested in small fluctuations around a given value of the phase and, in particular, we discuss the results regarding the estimation precision for $\phi=0$.

We consider a pure probe state $\varrho=\left|\psi_{0}\right\rangle\left\langle\psi_{0}\right|$, where

$$
\left|\psi_{0}\right\rangle=D(\alpha) S(\xi)|0\rangle
$$

is a generic single-mode pure Gaussian state, $S(\xi)=$ $\exp \left\{\xi a^{2}-\xi^{*} a^{\dagger 2}\right\}$ is the squeezing operator, $\xi=r e^{i \theta}$, and $\{\alpha, r, \theta\} \in \mathbb{R}$. As we consider the effect of phase rotation over a single-mode state, we are implying that we already have, 
as an implicit resource, a reference beam (typically a strong coherent state), such that the phase rotation is well defined with respect to this reference, and relative phases between terms with different photon number become observable. We then focus our attention on the behavior of the QFI as a function of the energy of the input state $\left|\psi_{0}\right\rangle$. A useful reparametrization corresponds to considering the three parameters $\left\{n_{0}, \beta, \theta\right\} \in \mathbb{R}$, where

$$
n_{0}=\left\langle\psi_{0}\left|a^{\dagger} a\right| \psi_{0}\right\rangle=\alpha^{2}+\sinh ^{2} r
$$

is the average number of photons in the probe state, and

$$
\beta=\frac{\sinh ^{2} r}{n_{0}}
$$

is its squeezing fraction (for $\beta=0$ the probe state is a coherent state, while for $\beta=1$ it is a squeezed vacuum state). Our main goal is to derive the maximum value of QFI for an input Gaussian state at fixed number of photons $n_{0}$, by optimizing it over the parameters $\theta$ and $\beta$. The QFI for a generic state $U_{\phi, \eta}\left|\psi_{0}\right\rangle$ can be evaluated analytically by means of either the formula in Eq. (9) or the one in Eq. (14). Its maximization over the squeezing angle yields $\theta_{\mathrm{opt}}=0$, which corresponds to squeezing of the $Q$ quadrature, while the numerical optimization over the squeezing fraction yields $\beta_{\text {opt }}=1$, indicating that the optimal strategy is to use all the photons to prepare a squeezed vacuum state. These results can be understood by observing that, for small values of $\phi$, the evolution $U_{\phi, \eta}$ corresponds to a phase-space displacement along the negative $P$ axis, followed by a phase rotation depending on $\phi$. Amplitude squeezing thus represents the best resource in order to detect the parameter $\phi$. In particular, the maximized QFI for $\phi=0$ reads

$$
H=8 n_{0}\left(n_{0}+1\right)+\left(2 n_{0}+2 \sqrt{n_{0}\left(n_{0}+1\right)}+1\right) \eta^{2} .
$$

As expected, in the undisturbed case of $\eta=0$, one re-obtains the result derived by Monras in Ref. [32]. We also observe that the second positive term implies that the estimation is improved over the case of no disturbance. This apparently counterintuitive result can be understood by taking into account the fact that the additional term in the Hamiltonian does actually increase the output average number of photons, which reads $n_{\text {out }}=n_{0}+\eta^{2}$. Because the additional energy is used as a resource for estimating the phase $\phi$, it is more interesting to consider the behavior of the QFI $H$ as a function of $n_{\text {out }}$ in order to fairly discuss the scaling as a function of the number of photons. As we can see in Fig. 1, at fixed values of $n_{\text {out }}$, the QFI takes smaller values by increasing the disturbance parameter $\eta$. Remarkably, we also observe that the nonclassical quadratic scaling is still preserved, showing how the nonclassical resource (squeezing) is fundamental to get the ultimate estimation precision.

\section{Performance of homodyne detection}

We now examine the suitability of homodyne detection to estimate the phase in the case of unitary linear disturbance. Homodyne detection corresponds to projection over the eigenstates $\left\{\Pi_{\omega}(x)=\left|x_{\omega}\right\rangle\left\langle x_{\omega}\right|\right\}$ of the generalized quadrature operator $X_{\omega}=a e^{i \omega}+a^{\dagger} e^{-i \omega}$. The corresponding Fisher information is evaluated as in Eq. (2), where the conditional

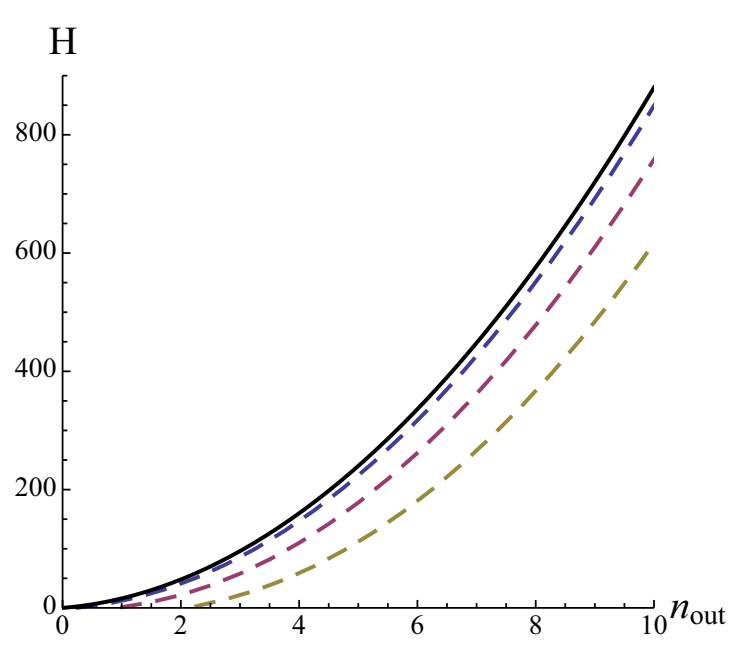

FIG. 1. (Color online) QFI, $H$, as a function of the output number of photons $n_{\text {out }}$. Solid line is the noiseless estimate $(\eta=0)$; dashed line is the estimate with unitary disturbance for different values of $\eta$. From top to bottom, $\eta=\{0.5,1,1.5\}$.

probability reads

$$
p(x \mid \phi)=\left|\left\langle x_{\omega}\left|U_{\phi, \eta}\right| \psi_{0}\right\rangle\right|^{2} .
$$

We consider a squeezed vacuum state which, as derived above, is the optimal probe in the case $\phi \approx 0$. We then optimize the FI over the homodyne angle $\omega$ and compare the result to the QFI by evaluating the ratio $F / H$. In Ref. [32] it was shown that, in the case of no disturbance, homodyne detection is optimal because the corresponding FI is equal to the QFI. As we can see in Fig. 2(a), at fixed disturbance parameter $\eta$, homodyne detection ceases to be optimal for probes with nonzero photon number, but it is nearly optimal when the probe is very weak ( $\left.n_{0} \approx 0\right)$ or very strong $\left(n_{0} \gg \eta^{2}\right)$. The near-to-optimality for a weak probe can be understood by observing the fact that the output state is basically a coherent state (due to the disturbance in the unitary operator), and homodyne detection is optimal for phase estimation with a coherent state. Regarding input states with a large average photon number, the disturbance (a)

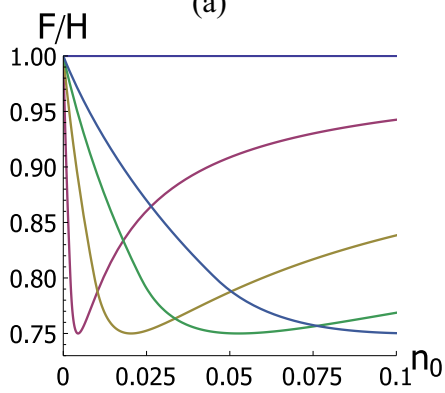

(b)

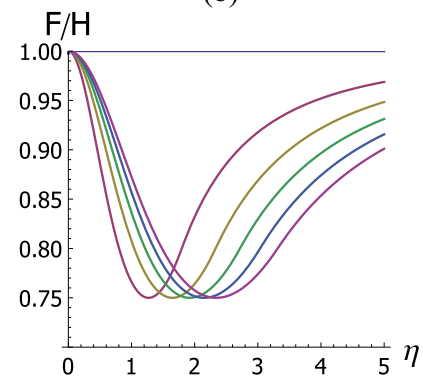

FIG. 2. (Color online) Ratio between the homodyne detection FI, $F$, and the corresponding QFI, $H$. (a) $F / H$ as function of the number of photons of the probe for different values of disturbance $\eta$; using the right ends of the curves as reference, from top to bottom: $\eta=$ $\{0.0,0.25,0.50,0.75,1.0\}$. (b) $F / H$ as function of the disturbance parameter for different values of the average number of photons of the probe; from top to bottom: $n_{0}=\{0,0.2,0.4,0.6,0.8,1.0\}$. 
can be considered as a small perturbation, and the noiseless optimality is recovered.

A symmetric description holds for the behavior of the ratio $F / H$ for fixed values of the average input photon number $n_{0}$ and as a function of the disturbance parameter $\eta$, which is plotted in Fig. 2 (right). Homodyne detection is indeed optimal for small and large values of $\eta$ (compared to the input photon number), corresponding in this case respectively to the situation where the disturbance can be considered as a small perturbation, or when the output state resembles a coherent state.

In Figs. 2(a) and 2(b) we also note that the ratio has a minimum which is always equal to $(F / H)_{\min }=3 / 4$, showing the overall efficiency of homodyne detection for the whole range of the parameters.

\section{RANDOM LINEAR DISTURBANCE}

We now turn our attention to a situation where the disturbance parameter is not fixed, being a random variable satisfying a (known) probability distribution. To keep the situation as general and symmetric as possible, we consider two disturbance operators in the Hamiltonian, leading to a displacement in phase space along orthogonal directions. In formula, we consider the unitary evolution

$$
U_{\phi, \eta}=\exp \left\{-i\left(\phi a^{\dagger} a+\eta_{1} Q+\eta_{2} P\right)\right\}
$$

where $\eta=\left(\eta_{1}, \eta_{2}\right)^{\top}$. If we consider the two disturbance parameters both distributed according to a Gaussian probability distribution centered at zero with the same variance $\Delta$, the average output (mixed) state reads

$$
\mathcal{G}_{\phi, \Delta}\left(\left|\psi_{0}\right\rangle\left\langle\psi_{0}\right|\right)=\int_{\mathbb{R}^{2}} d \eta_{1} d \eta_{2} \frac{e^{-\frac{\eta_{1}^{2}+\eta_{2}^{2}}{2 \Delta^{2}}}}{2 \pi \Delta^{2}} U_{\phi, \eta}\left|\psi_{0}\right\rangle\left\langle\psi_{0}\right| U_{\phi, \eta}^{\dagger} .
$$

We still consider as an input a generic pure Gaussian state, $\left|\psi_{0}\right\rangle=D(\alpha) S(\xi)|0\rangle$. In absence of the additional phase rotation, this channel is usually referred to as Gaussian noise $[35,38]$ and has been widely studied in the context of quantum communications [39-41]. From a physical point of view, this kind of evolution is relevant, for example, in the following two scenarios: (i) this evolution is indeed a good approximation for a bosonic field interacting with a high temperature bosonic bath, i.e., when spontaneous emission can be neglected; (ii) when a displacement operator is engineered on an input state, by mixing it with a coherent state at a beam splitter with low transmissivity [42], Gaussian noise on the output state is inherited from the amplitude and phase fluctuations of the laser which provides the ancillary coherent state.

As its effect is to displace the state incoherently in different directions of phase space with random amplitude, the output state will become mixed similar to a state in a phase-diffusion channel. However we can observe at least two main differences between this noisy channel and phase diffusion: the latter corresponds to a random phase rotation, i.e., one has a single disturbance operator $A=a^{\dagger} a$; as a consequence the action of the channel commutes with the phase rotation itself, and one can consider the two evolutions separately. Moreover, the state after a phase-diffusion channel will have the same amount of

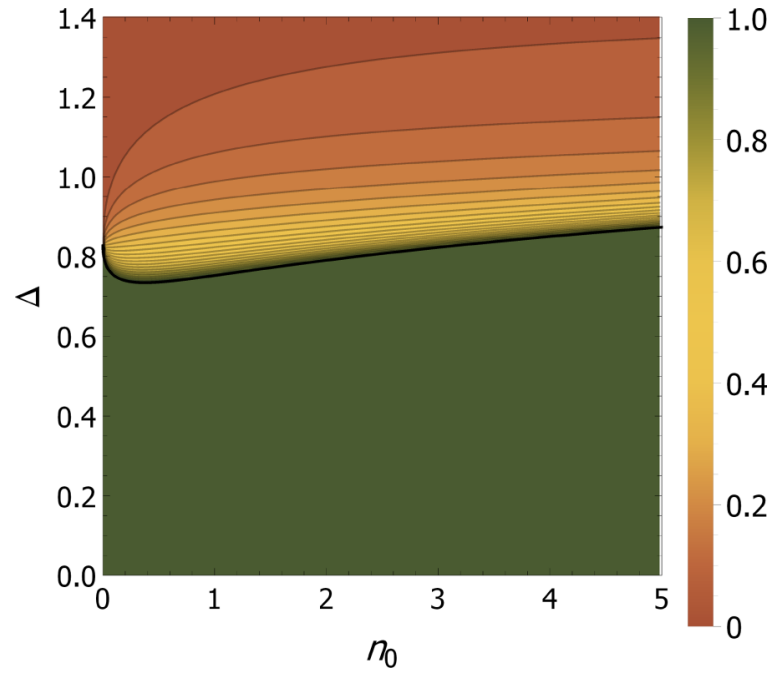

FIG. 3. (Color online) Optimal squeezing fraction $\beta_{\text {opt }}$ as function of the noise parameter $\Delta$ and number of photons $n_{0}$ in the probe. The threshold $\Delta_{c}\left(n_{0}\right)$ is represented with a black curve.

mean photon number as the input state, while after the channel described in Eq. (23), the output state will have an output average photon number $n_{\text {out }}=n_{0}+2 \Delta^{2}$. It is straightforward to see that the state remains Gaussian and, consequently, one can evaluate the QFI by using the formulas presented in Sec. II B. As in the unitary-disturbance case, we focus on small fluctuations around the value $\phi=0$, and we optimize the input Gaussian state for a fixed number of photons $n_{0}$, over the squeezing angle $\theta$ and the squeezing fraction $\beta$. If the optimal probe has a nonzero displacement parameter $\alpha$, the optimal squeezing angle is $\theta_{\mathrm{opt}}=\pi$ (phase squeezing) while, due to the symmetry of the disturbance introduced, the QFI does not depend on $\theta$ when the state is prepared in a squeezed vacuum state. We now discuss the behavior of the optimal squeezing fraction $\beta_{\text {opt }}$ for a fixed average input photon number $n_{0}$, and varying the disturbance parameter $\Delta$, by observing the contour plot of Fig. 3. For values of $\Delta$ smaller than a threshold $\Delta_{t}\left(n_{0}\right)$ (the black line in Fig. 3), the optimal state is always a squeezed vacuum state, and we have $\beta_{\text {opt }}=1$ as in the noiseless case. By increasing $\Delta$, the optimal squeezing fraction drops to $\beta_{\mathrm{opt}}=0$ : in more detail, for very small values of $n_{0}$, when $\Delta$ crosses the limiting value $\Delta_{t}(0)=\frac{\sqrt{1+\sqrt{3}}}{2}$ the optimal state changes very abruptly from a squeezed vacuum to a coherent state. For larger values of $n_{0}$, there is a region in the parameter space $\left(n_{0}, \Delta\right)$ where the optimal state is a displaced squeezed vacuum state. By studying more carefully the noise threshold, we observe that it has a minimum $\Delta_{t, \text { min }} \approx 0.734$ at $n_{0} \approx 0.375$, below which the optimal probe is the squeezed vacuum state irrespective of the number of photons in the probe. Moreover, the corresponding function can be well approximated for $n_{0} \gtrsim 20$, as $\Delta_{t}\left(n_{0}\right) \sim \sqrt[6]{n_{0} / 16}$, showing how the threshold increases very slowly with the mean photon number. Remarkably, one also observes that, for a fixed $\Delta$, for large values of the input energy $n_{0}$ the optimal state is still a squeezed vacuum state, suggesting that squeezing is still a resource when the noise parameter is small compared to the mean photon number. The behavior of the optmized 
(a)
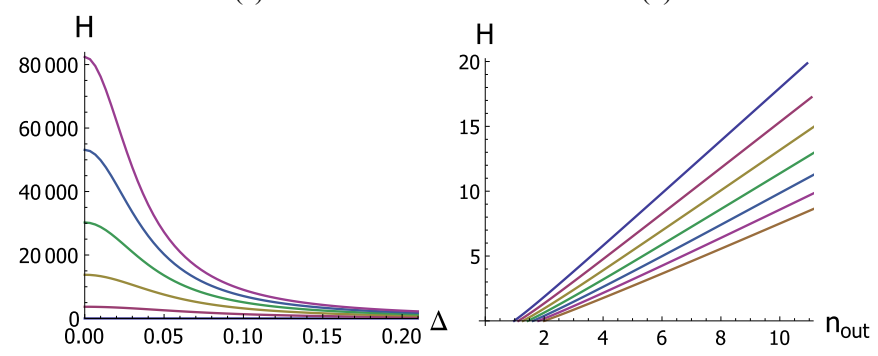

FIG. 4. (Color online) (a) Optimized QFI in the case of random linear disturbance as function of the noise parameter $\Delta$ for fixed values of the average photon number; from bottom left to top right $n_{0}=\{1,21,41,61,81,101\}$. (b) Optimized QFI as a function of the output photon number $n_{\text {out }}$ for fixed values of the noise parameter $\Delta$; from top to bottom $\Delta=\{0.7,0.75,0.8,0.85,0.9,0.95,1.0\}$.

QFI as a function of the noise parameter $\Delta$ is displayed in Fig. 4(a). Despite the fact that the energy of the output state increases for nonzero noise parameter, the ultimate estimation performances are degraded by the random linear disturbance. As in the previous case, we also plot the behavior of the QFI as a function of the output photon number $n_{\text {out }}$ in Fig. 4(b). We clearly observe that, in this case, the quadratic behavior is lost, and that the QFI scales linearly with $n_{\text {out }}$. Remarkably we can also compute an approximate value for the linear coefficient, obtaining

$$
H \approx a n_{\mathrm{out}}, \quad \text { with } a \approx \frac{1}{\Delta^{2}} .
$$

The approximation is more accurate for large values of the photon number, where the optimal probe state is more likely to be in a squeezed vacuum state. As expected, smaller values of $\Delta$ correspond to larger values of the coefficient and thus to larger values of the QFI; However, we observe that any nonzero values of the noise parameter are enough to lose the nonclassical scaling. This discontinuity in the scaling of the QFI is quite typical in noisy quantum metrology as it has been indeed widely observed in many different metrological problems $[13,20]$.

\section{Performance of homodyne detection}

As in the previous section we discuss the efficiency of homodyne detection for a random linear disturbance channel. The corresponding FI can be calculated by starting from the conditional probability

$$
p(x \mid \phi)=\left\langle x_{\omega}\left|\mathcal{G}_{\phi, \Delta}\left(\left|\psi_{0}\right\rangle\left\langle\psi_{0}\right|\right)\right| x_{\omega}\right\rangle .
$$

As before, we optimize over the homodyne angle $\omega$ and evaluate the ratio $F / H$ between the optimized FI and the QFI.

Before focusing on the optimal input states identified in the previous section, we study the optimality of homodyne detection for the two extreme cases; that is, for input squeezed vacuum states $(\beta=1)$ and coherent states $(\beta=0)$. Regarding the first case, i.e., $\left|\psi_{0}\right\rangle=S(r)|0\rangle$, the ratio $F / H$ is plotted in Fig. 5. If we fix the value of the input photon number $n_{0}=$ $\sinh ^{2} r$, homodyne detection results to be (nearly) optimal only for small values of the noise parameter $\Delta$, and the ratio $F / H$ decreases monotonically to the asymptotic value $F / H \approx 0.5$

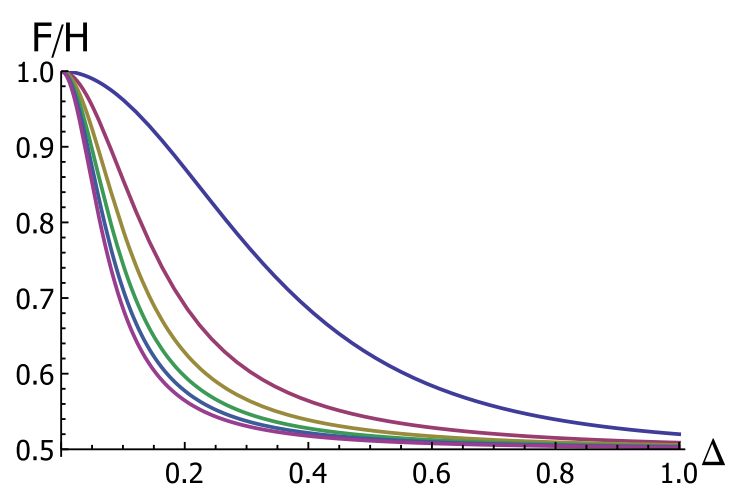

FIG. 5. (Color online) Ratio between the Fisher Information of the homodyne detection and the corresponding QFI for squeezed vacuum input states that have undergone a random linear disturbance. The ratio is plotted as a function of the noise parameter $\Delta$ and for different values of the average number of photons of the probe. From top to bottom: $n_{0}=\left\{10^{-9}, 2,4,6,8,10\right\}$.

by increasing $\Delta$. If we rather consider coherent states as the input, one can easily obtain that homodyne detection is always optimal for every value of the input energy and the noise parameter $\Delta$.

Having in mind these results for the extreme cases, we can now discuss the optimality of homodyne detection where we consider the optimal input state for each value of the noise parameter $\Delta$ and of the input average photon number $n_{0}$. It is useful to compare the corresponding ratio $F / H$, plotted in Fig. 6, with the plot of the optimal squeezing fraction in Fig. 3, which we discussed previously in this section. If we fix the value of the input photon number $n_{0}$ and vary the noise parameter $\Delta$, we observe the following behavior: the homodyne measurement is optimal for $\Delta \approx 0$, and its

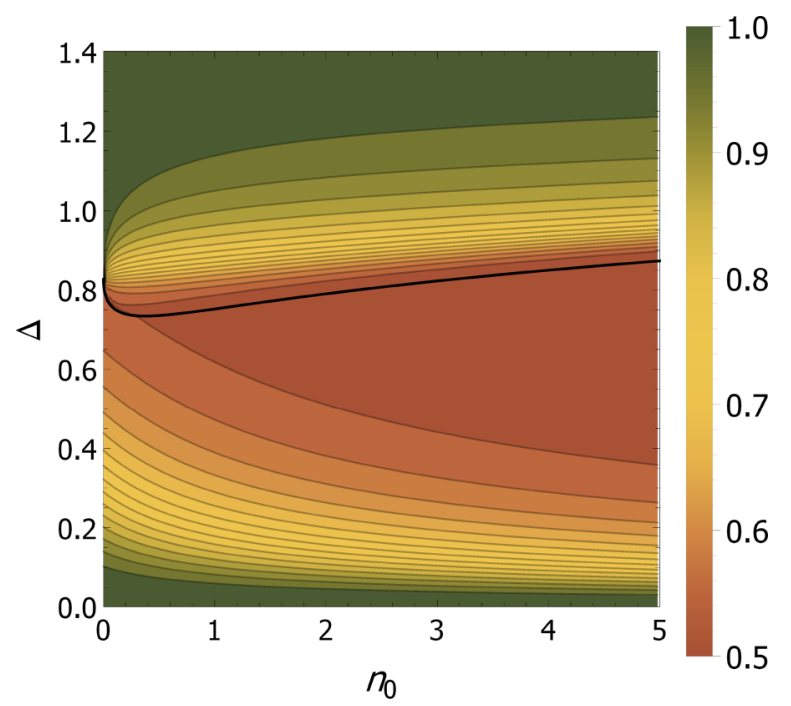

FIG. 6. (Color online) Ratio $F / H$ between the homodyne's FI over the QFI for optimized input state as a function of the input photon number $n_{0}$ and of the noise parameter $\Delta$. The superimposed black line corresponds to the input state threshold $\Delta_{t}\left(n_{0}\right)$ which divides regions with squeezed vacuum probes $\left[\Delta<\Delta_{t}\left(n_{0}\right)\right]$ and displaced squeezed probes $\left[\Delta>\Delta_{t}\left(n_{0}\right)\right]$. 
efficiency decreases reaching a minimum for a certain value of $\Delta$. The ratio $F / H$ starts to increase with $\Delta$, reaching values near to unity for large values of $\Delta$. In particular, we observe that the minimum of the ratio $F / H$ occurs in proximity of the threshold $\Delta_{t}\left(n_{0}\right)$ described in the previous section, which divides the different regions of parameters for the optimal squeezing fraction. In Fig. $6, \Delta_{t}\left(n_{0}\right)$ is depicted as a superimposed black line. In the region below the black line $\left[\Delta<\Delta_{t}\left(n_{0}\right)\right]$, the optimal input state is a squeezed vacuum, and the behavior of the ratio $F / H$ does indeed correspond to the plots in Fig. 5. In the region above the black line $\left[\Delta>\Delta_{t}\left(n_{0}\right)\right]$, the optimal state is a displaced squeezed state, which tends to a coherent state for larger noise. As a consequence the ratio $F / H$ starts to increase by increasing $\Delta$, reaching eventually the optimality which, as discussed above, is always obtained for input coherent states.

\section{CONCLUSIONS}

The effect of noise and imperfection on the performances of quantum metrology protocols has received a lot of attention since the seminal paper by Heulga et al. [12], leading to the very general results obtained recently in Refs. [13,14]. In this paper, we discussed the case where an unwanted term is present in the Hamiltonian, generating the phase shift that one wants to estimate. In particular, we considered both unitary and random linear disturbance, with an input Gaussian state, optimizing over the squeezing fraction and the squeezing angle. While in the case of unitary disturbance, the squeezed vacuum is shown to be the optimal probe state and the nonclassical quadratic scaling of the QFI is still observed, in the presence of random disturbance the optimal squeezing fraction crucially depends on the input energy and on the noise parameter values and, more importantly, any nonzero value of the noise is enough to cause a linear scaling between the QFI and the number of photons of the quantum state.

We also discussed the performance of homodyne detection, which is shown to be in general an efficient measurement in both cases, despite the fact that the optimality is observed only in some regions of the parameter space characterizing the input state and the noisy channel.

\section{ACKNOWLEDGMENTS}

D.D.S. acknowledges support from the Brazilian funding agencies CNPq, through Grant 237095/2012-2, and FAPESP, through Grant 2011/00220-5. D.D.S. is also thankful to the CQD theory group at the Imperial College London for the warm hospitality. M.G.G. acknowledges support from UK EPSRC through Grant EP/K026267/1. M.S.K. thanks the UK EPSRC for financial support.
[1] V. Giovannetti, S. Lloyd, and L. Maccone, Nat. Photonics 5, 222 (2011).

[2] M. J. Holland and K. Burnett, Phys. Rev. Lett. 71, 1355 (1993).

[3] J. J. Bollinger, W. M. Itano, D. J. Wineland, and D. J. Heinzen, Phys. Rev. A 54, R4649(R) (1996).

[4] P. Kok, H. Lee, and J. P. Dowling, Phys. Rev. A 65, 052104 (2002).

[5] V. Giovannetti, S. Lloyd, and L. Maccone, Science 306, 1330 (2004).

[6] V. Giovannetti, S. Lloyd, and L. Maccone, Phys. Rev. Lett. 96, 010401 (2006).

[7] T. Nagata, R. Okamoto, J. L. O’Brien, K. Sasaki, and S. Takeuchi, Science 316, 726 (2007).

[8] H. Yonezawa, D. Nakane, T. A. Wheatley, K. Iwasawa, S. Takeda, H. Arao, K. Ohki, K. Tsumura, D. W. Berry, T. C. Ralph, H. M. Wiseman, E. H. Huntington, and A. Furusawa, Science 337, 1514 (2012).

[9] S. Simmons, J. A. Jones, S. D. Karlen, A. Ardavan, and J. J. L. Morton, Phys. Rev. A 82, 022330 (2010).

[10] B. J. Luff, J. S. Wilkinson, J. Piehler, U. Hollenbach, J. Ingenhoff, and N. Fabricius, J. Lightwave Technol. 16, 583 (1998).

[11] A. Crespi, M. Lobino, J. C. F. Matthews, A. Politi, C. R. Neal, R. Ramponi, R. Osellame, and J. L. O’Brien, Appl. Phys. Lett. 100, 233704 (2012).

[12] S. F. Huelga, C. Macchiavello, T. Pellizzari, A. K. Ekert, M. B. Plenio, and J. I. Cirac, Phys. Rev. Lett. 79, 3865 (1997).

[13] R. Demkowicz-Dobrzanski, J. Kolodynski, and M. Gută, Nat. Commun. 3, 1063 (2012).
[14] B. M. Escher, R. L. de Matos Filho, and L. Davidovich, Nat. Phys. 7, 406 (2011).

[15] R. Chaves, J. B. Brask, M. Markiewicz, J. Kolodynski, and A. Acin, Phys. Rev. Lett. 111, 120401 (2013).

[16] W. Dur, M. Skotiniotis, F. Frowis, and B. Kraus, Phys. Rev. Lett. 112, 080801 (2014).

[17] G. Arrad, Y. Vinkler, D. Aharonov, and A. Retzker, Phys. Rev. Lett. 112, 150801 (2014).

[18] E. M. Kessler, I. Lovchinsky, A. O. Sushkov, and M. D. Lukin, Phys. Rev. Lett. 112, 150802 (2014).

[19] A. W. Chin, S. F. Huelga, and M. B. Plenio, Phys. Rev. Lett. 109, 233601 (2012).

[20] R. Demkowicz-Dobrzanski, U. Dorner, B. J. Smith, J. S. Lundeen, W. Wasilewski, K. Banaszek, and I. A. Walmsley, Phys. Rev. A 80, 013825 (2009).

[21] M. Kacprowicz, R. Demkowicz-Dobrzanski, W. Wasilewski, K. Banaszek, and I. A. Walmsley, Nat. Photonics 4, 357 (2010).

[22] P. J. D. Crowley, A. Datta, M. Barbieri, and I. A. Walmsley, Phys. Rev. A 89, 023845 (2014).

[23] S. I. Knysh, V. N. Smelyanskiy, and G. A. Durkin, Phys. Rev. A 83, 021804 (2011).

[24] D. Brivio, S. Cialdi, S. Vezzoli, B. T. Gebrehiwot, M. G. Genoni, S. Olivares, and M. G. A. Paris, Phys. Rev. A 81, 012305 (2010).

[25] M. G. Genoni, S. Olivares, and M. G. A. Paris, Phys. Rev. Lett. 106, 153603 (2011).

[26] M. G. Genoni, S. Olivares, D. Brivio, S. Cialdi, D. Cipriani, A. Santamato, S. Vezzoli, and M. G. A. Paris, Phys. Rev. A 85, 043817 (2012). 
[27] B. M. Escher, L. Davidovich, N. Zagury, and R. L. de Matos Filho, Phys. Rev. Lett. 109, 190404 (2012).

[28] S. I. Knysh and G. A. Durkin, arXiv:1307.0470.

[29] S. I. Knysh, E. H. Chen, and G. A. Durkin, arXiv:1402.0495.

[30] M. D. Vidrighin, G. Donati, M. G. Genoni, X. M. Jin, W. S. Kolthammer, M. S. Kim, A. Datta, M. Barbieri, and I. A. Walmsley, Nat. Commun. 5, 3532 (2014).

[31] A. De Pasquale, D. Rossini, P. Facchi, and V. Giovannetti, Phys. Rev. A 88, 052117 (2013).

[32] A. Monras, Phys. Rev. A 73, 033821 (2006).

[33] C. W. Helstrom, Quantum Detection and Estimation Theory (Academic Press, New York, 1976).

[34] M. G. A. Paris, Int. J. Quantum Inform. 7, 125 (2009).
[35] A. Ferraro, S. Olivares, and M. G. A. Paris, Gaussian States in Quantum Information (Bibliopolis, Napoli, 2005).

[36] A. Monras, arXiv:1303.3682.

[37] O. Pinel, P. Jian, N. Treps, C. Fabre, and D. Braun, Phys. Rev. A 88, 040102 (2013).

[38] N. J. Cerf, Gerd Leuchs, and E. S. Polzik, Quantum Information with Continuous Variables of Atoms and Light (Imperial College Press, London, 2007), pp. 23-42.

[39] A. S. Holevo and R. F. Werner, Phys. Rev. A 63, 032312 (2001). [40] J. Harrington and J. Preskill, Phys. Rev. A 64, 062301 (2001).

[41] V. Giovannetti, S. Lloyd, L. Maccone, J. H. Shapiro, and B. J. Yen, Phys. Rev. A 70, 022328 (2004).

[42] M. G. A. Paris, Phys. Lett. A 217, 78 (1996). 